POS $\quad$ PROCEEDINGS

\title{
The VHE gamma-ray periodicity of PG1553+113: a possible probe of a system of binary supermassive black hole
}

\author{
Elisa Prandini* \\ Astronomy Department University of Geneva \& ETH Zurich Institute for Particle Physics \\ E-mail: elisa.prandini@unige.ch
}

Antonio Stamerra

INAF, Torino

Simona Paiano

INAF \& INFN, Padova

Paolo Da Vela

INFN, Pisa

Josefa Becerra Gonzalez

NASA Goddard Space Flight Center

Gareth Hughes,

ETH Zurich, Institute for Particle Physics

Elina Lindfors

Tuorla Observatory

Ulisses Barres de Almeida

Centro Brasileiro de Pesquisas Fisicas, Rio de Janeiro

Stefano Covino, Giacomo Bonnoli

INAF, Brera

Angela Sandrinelli

University of Insubria, Como

Angelo Antonelli

INAF, Roma

for the MAGIC Collaboration 
The blazar PG1553+113 is an active galaxy with uncertain redshift detected at very high energies (VHE; E > $100 \mathrm{GeV}$ ) both during high and quiescent states. We have observed with the MAGIC telescopes from La Palma PG 1553+113 at VHE since 2005, making this blazar one of the best studied MAGIC sources.

Recently, the Fermi/LAT collaboration has reported the detection of a hint of a 2-year periodicity in the integral flux emitted by the source both at high energy gamma rays $(\mathrm{E}>100 \mathrm{MeV})$ and at optical wavelengths. Remarkably, this periodicity, if confirmed, might be interpreted as an evidence of the presence of a binary supermassive black hole system in the nucleus of PG1553+113. In this contribution, we present the result of our analysis of 10 years of PG 1553+113 MAGIC data. In particular, we test the hypothesis of a periodic modulation of the overall emitted flux at VHE, search for evidences of correlation with the emission detected at other wavelengths, and critically discuss our findings in the framework of the binary supermassive black hole model.

The 34th International Cosmic Ray Conference,

30 July- 6 August, 2015

The Hague, The Netherlands

${ }^{*}$ Speaker. 